\title{
EDITORIAL
}

nature

cell biology

\section{Review recycling}

\section{Thirty neuroscience journals share referee reports.}

Biologists aim for publication in the highest ranked journal possible to maximize the visibility and citations of their research, but also because the publication venue can directly affect funding. As a result, papers often pass through several journals before being accepted, leading to redundant peer reviewing and delays to publication.

How can we smooth the road to publication? First, choose carefully where to submit. The pressures to publish prestigiously are considerable, but nothing is more disheartening than multiple rejections, with the constant risk of being scooped. Consider the scope and quality of your chosen journal and obtain feedback from colleagues peripheral to the work. Second, do not submit premature work, so as to 'allow' referees to ask for obvious experiments (equally, referees must not feel obliged to ask for more experiments; see April 2008 Editorial). A rejection may not be due to fundamental reservations about the data, but because it is not sufficiently developed or is editorially inappropriate.

A constructive referee report can serve to improve the quality of a rejected manuscript. Nevertheless, sharing referee reports between journals would certainly reduce redundancy in the system. For many years, the Nature journals have shared referee reports: after rejection, authors can submit a revised manuscript to another title and full reports from all previous rounds of review and referee identities are transferred automatically. Of course, authors can choose not to reveal the submission history by submitting de novo. Editors use transferred reports at their discretion and may complement them with further expert advice - this is usually in the authors' interests as Nature journals share similar standards.

The neuroscience community has gone one step further: in January a group of editors set up the Neuroscience Peer Review Consortium (http://nprc.incf.org) with support from the Society for Neuroscience. As with Nature journal transfers, an author can choose to transfer a rejected manuscript with referee reports between one of currently thirty other neuroscience journals. Remarkably, this project encompasses a good fraction of the neuroscience literature with a wide spectrum of journal rankings. Referees are asked for permission to release their identity and confidential comments are not transferred. This is a promising scheme, but we note a number of potential challenges: 1) transfers can cross multiple journal tiers and it will be a challenge to extract relevant information from reports intended for a different journal; however, referees can add recommendations beyond the target journal; 2) higher tier journals may be swamped with submissions although, so far, Nature Neuroscience has only transferred four manuscripts; 3) journal formats differ (this does not affect consideration at this journal); 4) the prospect of being contacted by multiple journals may discourage referees. Interestingly, only one of twelve referees at Nature Neuroscience asked for their identity to be withheld and feedback from referees has been encouraging; 5) consortium journals are committed to preserving referee confidentiality, but this will be a challenge given the large editorial boards of many journals.

None of these issues presents insurmountable obstacles, as authors retain control and decide whether a second submission with the same reports is beneficial. Would this work for a diverse collection of fields such as molecular cell biology? The neurosciences community is certainly sizeable but also more homogeneous. This is a worthy experiment and it will be interesting to see how successful it proves to be after the current one-year trial.

\section{Open access archiving}

\section{The Nature journals upload manuscripts to PubMed Central on request.}

Scientific journal publishing will at first sight seem bizarre to those accustomed to other forms of publishing: scientists critique their peers' work for free, often resulting in dramatic improvements to their papers. Journals do not pay their authors, but instead often charge publication fees to defer production costs. Many charge for subscriptions to keep submission fees affordable. How is this apparently topsy-turvy world possible? The reason is, of course, that peer-reviewed papers represent the validated measure of a researcher's output, which directly affects funding and career progression. Referees graciously provide their services because they see the value in community-based assessment of research output and they know that their research also benefits from good peer review. Authors and readers generally appreciate that papers benefit from a thorough editorial process that improves accessibility of specialized data and concepts. Equally, they understand that this process incurs significant costs. However, it is debatable whether copyright protection should extend to publishers. Since 2002, authors retain copyright at the Nature journals but grant them an exclusive licence to publish their work. Nevertheless, a constructive grass roots movement has emerged that argues for open access to all primary scientific data. Much has been written about the merits of various publishing models and indeed most permutations are now being subjected to real-world testing. Suffice it to say that 'open access' should apply to both reader and author, as excessive submission or publication charges can become prohibitive to all but the best funded laboratories.

PubMed Central (PMC), an open access archive set up by the US National Library of Medicine and its European counterpart UKPMC, provides a valuable repository for published work and is endorsed by many key funding bodies. NPG has encouraged archiving of accepted papers at PMC (or other appropriate repositories or websites) since 2005. The challenge is to make scientific literature accessible while recognizing the need to finance the publishing process. For this reason, the accepted, rather than the copyedited manuscripts, are uploaded six months after publication. The rationale is that the journal remains the formal point of reference, whereas PMC provides essential information centrally and at no cost. Links from PMC provide seamless access to the enhanced journal article.

As this dual publication approach gains momentum, NPG has launched an automated free service at the Nature journals for authors whose funding bodies require PMC posting. All it takes is to opt in at the submission stage (see www.nature.com/authors/author_services/ deposition.html). Before posting, PMC confirms the deposition with the authors, and manuscripts are uploaded after six months. In the future, this will be extended to other NPG journals and repositories. 